\title{
Neurological comorbidity and severity of COVID-19
}

\author{
Alberto Romagnolo ${ }^{1}$. Roberta Balestrino ${ }^{1}$ - Gabriele Imbalzano ${ }^{1}$. Giovannino Ciccone ${ }^{2}$. Franco Riccardini ${ }^{3}$. \\ Carlo Alberto Artusi ${ }^{1} \cdot$ Marco Bozzali $^{1,4}$. Bruno Ferrero ${ }^{1}$. Elisa Montalenti ${ }^{1}$. Elisa Montanaro ${ }^{1}$. \\ Mario Giorgio Rizzone ${ }^{1} \cdot$ Giovanna Vaula ${ }^{1}$. Maurizio Zibetti ${ }^{1} \cdot$ Leonardo Lopiano $^{1}$
}

Received: 8 June 2020 / Revised: 22 July 2020 / Accepted: 27 July 2020 / Published online: 4 August 2020

(c) The Author(s) 2020

\begin{abstract}
Objective Neurological symptoms of COVID-19 patients have been recently described. However, no comprehensive data have been reported on pre-existing neurological comorbidities and COVID-19. This study aims at evaluating the prevalence of neurological comorbidities, and their association with COVID-19 severity.

Methods We evaluated all consecutive patients admitted to the Emergency Room (ER) of our hospital between the 3rd March and the 14th April 2020, and diagnosed with COVID-19. Data on neurological and non-neurological diseases were extracted, as well as data on demographic characteristics and on severity degree of COVID-19. The prevalence of neurological comorbidities was calculated, and multivariate binary logistic regression analyses were used to estimate the association between neurological diseases and COVID-19 severity.

Results We included 344 patients. Neurological comorbidities accounted for $22.4 \%$ of cases, with cerebrovascular diseases and cognitive impairment being the most frequent. Neurological comorbidity resulted independently associated with severe COVID-19 (OR 2.305; $p=0.012)$, as well as male gender ( $p=0.001)$, older age $(p=0.001)$, neoplastic diseases $(p=0.039)$, and arterial hypertension $(p=0.045)$. When neurological comorbidity was associated with non-neurological comorbidities, the OR for severe COVID-19 rose to $7.394(p=0.005)$. Neurological patients, in particular cerebrovascular and cognitively impaired ones, received more respiratory support indication.

Conclusion Neurological comorbidities represent a significant determinant of COVID-19 severity, deserving a thorough evaluation since the earliest phases of infection. The vulnerability of patients affected by neurological diseases should suggest a greater attention in targeting this population for proactive viral screening.
\end{abstract}

Keywords COVID-19 $\cdot$ Neurology $\cdot$ Stroke $\cdot$ Cerebrovascular disease $\cdot$ Dementia

Leonardo Lopiano

leonardo.lopiano@unito.it

1 Department of Neuroscience "Rita Levi Montalcini", University of Turin, Via Cherasco 15, 10126 Turin, Italy

2 Section of Clinical Epidemiology, Center for Epidemiology and Oncologic Prevention (CPO Piemonte), Città della Salute e della Scienza, Turin, Italy

3 Department of Medical Sciences, Front Line P.S. P.O. Molinette, Città Della Salute e Della Scienza, Turin, Italy

4 Department of Neuroscience, Brighton\& Sussex Medical School, University of Sussex, Brighton, East Sussex, UK

\section{Introduction}

Coronavirus disease 2019 (COVID-19) is an infectious disease caused by severe acute respiratory syndrome coronavirus-2 (SARS-CoV-2), declared a pandemic on 11 March 2020 [1]. Clinical features range from the absence of symptoms to severe respiratory failure [2]. A rapidly increasing number of articles have been published on COVID-19, including numerous reports and studies on associated neurological symptoms and complications [3], such as acute stroke [4], hyposmia [5], Guillain-Barrè syndrome [6], encephalitis [7]. It has been shown that about one-third of COVID-19 patients develop neurological symptoms [3, 8], in most cases associated with a more severe infection [3], indicating a potential neurotropism of SARS-CoV-2 as one of the possible mechanisms of neurological damage [9, 
10]. A recent retrospective study reported that inpatients from a neurological ward affected by COVID-19 showed a worse outcome compared to those infection-free [11]. However, no data have been reported yet on the prevalence and the association with infection severity of pre-existing neurological comorbidities in COVID-19 patients. A scoping review on the occurrence of neurological diseases in COVID-19 patients reported a prevalence of about $8 \%$ [12]. Nevertheless, this review highlighted the methodological heterogeneity in the reviewed papers, which limit a reliable estimation of prevalence of neurological diseases in COVID19 patients, as well as their association with the infection severity.

The aim of our study was to evaluate, on a large cohort of consecutive patients admitted to the Emergency Room (ER) and diagnosed with COVID-19, the prevalence of neurological comorbidities and their possible association with a more severe form of COVID-19 onset.

\section{Methods}

We evaluated all consecutive patients admitted to the ER of the "Città della Salute e della Scienza di Torino Hospital" between 3 March 2020 and 14 April 2020, and diagnosed with COVID-19 by means of a positive Reverse Transcription Polymerase Chain Reaction nasopharyngeal swab.

\section{Comorbidities definition}

In all patients, we assessed both neurological and non-neurological comorbidities. A medical condition was attributed to the patients when: (a) defined diagnosis, and/or (b) unequivocal diagnostic test results, and/or (c) specific medical/surgical treatment, and/or (d) specific follow-up were reported. Moreover, previous clinical notes available in our hospital electronic archives were reviewed.

The Charlson Comorbidity Index (CCI) [13], defined as the sum of the weighted scores of various comorbid conditions (Table 1), was calculated for each patient to grade their comorbid conditions.

\section{Infection severity definition}

The severity of COVID-19 at the time of admission to ER was evaluated by means of the 2007 Infectious Diseases Society of America/American Thoracic Society Criteria for Defining Severe Community-acquired Pneumonia [14]. Severe disease was identified in patients presenting with one major criterion or three or more minor criteria (Table 2).
Table 1 Components and relative weights of the Charlson Comorbidity Index

\begin{tabular}{ll}
\hline Weight & Comorbidities \\
\hline $1 \times$ & Myocardial infarction \\
& Congestive heart failure \\
& Peripheral vascular disease \\
& Cerebrovascular disease \\
& Dementia \\
& Chronic obstructive pulmonary disease \\
& Connective tissue disease \\
& Ulcer disease \\
& Mild liver disease \\
& Diabetes mellitus \\
& Hemiplegia \\
& Moderate/severe renal disease \\
& Diabetes with end-stage organ damage \\
& Any tumor \\
& Leukemia \\
& Lymphoma \\
& Moderate/severe liver disease \\
& Metastatic solid tumor \\
& AIDS \\
\hline & \\
\hline &
\end{tabular}

The Charlson Comorbidity Index results by the sum of the various comorbidities multiplied by their weight score

Table 22007 Infectious Diseases Society of America/American Thoracic Society Criteria for Defining Severe Community-acquired Pneumonia

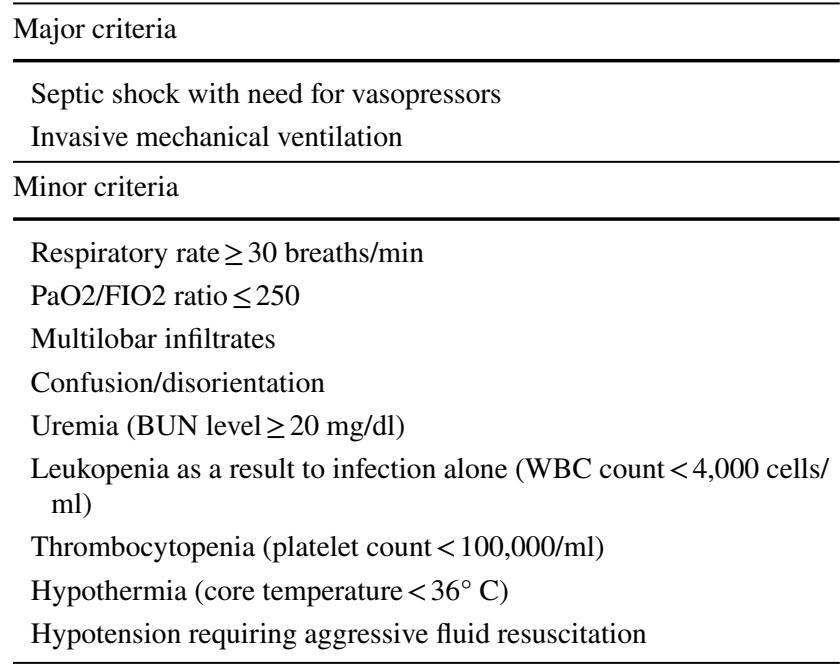

A severe form of infection was defined by the presence of either one major criterion or three or more minor criteria

$B U N$ blood urea nitrogen; $\mathrm{PaO} / \mathrm{FiO} 2$ arterial oxygen pressure/fraction of inspired oxygen, $W B C$ white blood cell 


\section{Discharge from ER definition}

Patients' discharge from the ER was classified as follows

(a) Home: discharge at home with indication to fiduciary isolation and health surveillance by the general practitioner;

(b) Internal Medicine Unit without mechanical respiratory support: admission to a ward equipped for best medical treatment and respiratory support with oxygen mask;

(c) Internal Medicine Unit with non-invasive mechanical respiratory support: admission to a ward equipped with non-invasive mechanical ventilation for respiratory support;

(d) Intensive Care Unit (ICU): admission to a ward equipped with invasive mechanical ventilation for respiratory support.

\section{Statistical analysis}

Descriptive statistics (mean, standard deviation, range) were used for continuous variables and frequency for categorical data. The Mann-Whitney test and Fisher's exact test were used for comparisons between groups, as appropriate. A first univariate binary logistic regression was used to estimate the odds ratio (OR) of presenting severe infection (dependent variable), considering as independent variables various demographic and clinical features, including neurological comorbidity. Then, we performed a second, multivariate, binary logistic regression analysis considering as independent variables every feature resulting significantly associated with severe infection in the previous univariate analysis. Finally, a third binary logistic regression analysis was performed, adjusting for age and CCI, considering four categories of patients as independent variable: (a) absence of any neurological or non-neurological disease (used as control group) (b) presence of neurological disease without other comorbidities, (c) presence of neurological disease plus other comorbidities, and (d) presence of comorbidities without neurological diseases. The Hosmer and Lemeshow's goodness-of-fit test was applied. In case of multiple comparisons, Bonferroni's correction was used. All $p$-values reported were two-tailed, and a $p<0.05$ was considered statistically significant. Data were analyzed using the Statistical Package for the Social Sciences (SPSS 22 for Mac, Chicago, IL).

\section{Ethics}

This study received approval from the ethical standards committee on human experimentation (Comitato Etico Interaziendale AOU Città della Salute e della Scienza di Torino, AO Ordine Mauriziano di Torino, ASL Città di Torino;
Protocol number 00172/2020, approved May 5th, 2020), and patients gave their written informed consent.

\section{Results}

The main demographic and clinical features of 344 consecutive patients evaluated are summarized in Table 3 .

Table 3 Demographic and Clinical features

\begin{tabular}{|c|c|}
\hline Gender (males/females) & $204 / 140(59.3 \% / 40.7 \%)$ \\
\hline Age (years) & $61.5 \pm 17.8(15-98)$ \\
\hline Time from symptoms onset (days) & $6.1 \pm 4.4(0-30)$ \\
\hline \multicolumn{2}{|l|}{ Symptoms at onset (\%) } \\
\hline Fever & $100(29.1 \%)$ \\
\hline Cough & $32(9.3 \%)$ \\
\hline Dyspnea & $17(4.9 \%)$ \\
\hline Anosmia & $7(2.0 \%)$ \\
\hline Myalgia/Asthenia & $6(1.7 \%)$ \\
\hline Sore throat & $5(1.5 \%)$ \\
\hline Confusion & $4(1.2 \%)$ \\
\hline Thoracic pain & $3(0.9 \%)$ \\
\hline Diarrhea & $2(0.6 \%)$ \\
\hline Conjunctivitis & $1(0.3 \%)$ \\
\hline Jaundice & $1(0.3 \%)$ \\
\hline Syncope & $1(0.3 \%)$ \\
\hline Fever and cough & $88(25.6 \%)$ \\
\hline Fever and dyspnea & $13(3.8 \%)$ \\
\hline Fever and diarrhea & $10(2.9 \%)$ \\
\hline Fever and anosmia & $5(1.5 \%)$ \\
\hline Cough and dyspnea & $5(1.5 \%)$ \\
\hline Fever and sore throat & $4(1.2 \%)$ \\
\hline Three or more symptoms & $40(11.6 \%)$ \\
\hline Neurological Diseases- $N(\%)$ & $77(22.4 \%)$ \\
\hline Arterial Hypertension $-N(\%)$ & $158(45.9 \%)$ \\
\hline Neoplastic Diseases-N $(\%)$ & $49(14.2 \%)$ \\
\hline Diabetes-N $-N)$ & $42(12.2 \%)$ \\
\hline $\begin{array}{l}\text { Chronic obstructive pulmonary disease- } \\
N(\%)\end{array}$ & $41(11.9 \%)$ \\
\hline Renal failure $-N(\%)$ & $18(5.2 \%)$ \\
\hline Charlson Comorbidity Index & $2.0 \pm 2.5(0-16)$ \\
\hline Institutionalized patients- $-N(\%)$ & $19(5.5 \%)$ \\
\hline Severe infection $-N(\%)$ & $118(34.3 \%)$ \\
\hline \multicolumn{2}{|l|}{ Discharge from Emergency Room-N $(\%)$} \\
\hline Home & $126(36.6 \%)$ \\
\hline $\begin{array}{l}\text { Internal Medicine Unit without mechani- } \\
\text { cal respiratory support }\end{array}$ & $170(49.4 \%)$ \\
\hline $\begin{array}{l}\text { Internal Medicine Unit with non-invasive } \\
\text { mechanical respiratory support }\end{array}$ & $25(7.3 \%)$ \\
\hline Intensive Care Unit & $23(6.7 \%)$ \\
\hline
\end{tabular}

Results are reported as average \pm standard deviation (range) or absolute values (percentage), as appropriate 
The mean age of the entire sample was 61.5 years: $4.7 \%$ of patients were $\leq 30$-year-old, while $33.7 \%$ were $\geq 70$-yearold. Male accounted for $59.3 \%$ of cases. The mean latency between the symptoms' onset and the ER admittance was 6.1 days, ranging from 0 to 30 days; only two patients ( $0.6 \%)$ with mild symptoms (sore throat and sporadic cough) had a latency of 30 days, while $91.6 \%$ of patients were evaluated within 10 days from the onset, and $7.8 \%$ between 11 and 15 days. The most frequent symptom at COVID-19 onset was fever $(74.1 \%$; $n=255$; isolated in 100 patients, associated with $\geq 1$ other symptoms in 155 patients), followed by cough $(44.8 \% ; n=154)$, dyspnea $(13.7 \% ; n=47)$, and diarrhea $(9.3 \% ; n=32)$. The majority of patients reported a monosymptomatic onset $(52.0 \% ; n=179)$, while $36.3 \%$ $(n=125)$ presented with two symptoms, and $11.6 \%(n=40)$ with three or more symptoms. Thirty-four percent of cases $(n=118)$ entered the ER with a severe form of infection.

Arterial hypertension was the most frequent comorbidity (45.9\%), followed by neurological diseases $(22.4 \%)$, neoplastic diseases (14.2\%), diabetes (12.2\%), chronic obstructive pulmonary disease (COPD) (11.9\%), and renal failure $(5.2 \%)$.

None of the patients were treated with specific antiviral drugs before admission; 9 of them (2.6\%) were on hydroxychloroquine therapy ( $200 \mathrm{mg}$ bid; therapy duration $5.2 \pm 2.6$ days, range $1-10)$.

\section{Prevalence and type of neurological diseases}

A total of $22.4 \%$ of patients $(n=77)$ showed a neurological comorbidity. Compared to patients not affected by neurological diseases (Table 4), they were disproportionately overrepresented among the severe COVID-19 $(p<0.001)$. They were older (mean difference 16.2 years; $\mathrm{p}<0.001$ ), had a shorter interval between symptoms onset and ER admittance (mean difference 1.9 days; $p=0.001$ ), and were more frequently affected by hypertension, renal failure, and neoplastic diseases $(p \leq 0.001)$; their CCI was higher $(p<0.001)$, and they presented a higher prevalence of institutionalization $(p<0.001)$. Pre-existing cerebrovascular diseases were the most common comorbidity, affecting $39.0 \%$ of patients ( $n=30 / 77$, including 7 patients with hemorrhagic and 23 with ischemic stroke), followed by cognitive impairment (32.5\%, including 4 patients with Mild Cognitive Impairment, 10 with Alzheimer's Disease or Alzheimer's Diseaselike dementia, and 11 with vascular dementia), migraine or chronic tension-type headache or trigeminal neuralgia $(14.3 \% ; n=11)$, epilepsy $(6.5 \% ; n=5)$, peripheral neuropathy $(5.2 \% ; n=4)$, Parkinson disease $(1.3 \% ; n=1)$, and multiple sclerosis $(1.3 \% ; n=1)$.

A minority of patients $(18.2 \% ; n=14)$ suffered only from neurological disease. The remaining 63 patients suffered also from one $(32.5 \% ; n=25)$, two $(37.7 \% ; n=29)$, or three or more $(11.7 \% ; n=9)$ other comorbidities, with arterial hypertension representing the most frequent comorbidity (90.5\% of cases), followed by neoplastic diseases (36.5\% of cases).

\section{Neurological diseases and infection severity}

The univariate binary logistic regression analysis (Table 5) revealed that a more severe form of infection was significantly associated with the presence of neurological disease (OR 5.855; 95\% CI 3.387-10.122; $p=0.001$ ), together with male gender, arterial hypertension, diabetes, renal failure, COPD, neoplastic disease, institutionalization, older age, and higher CCI.

On the multivariate binary logistic regression analysis (Fig. 1a), the presence of neurological diseases remained independently associated with severe infection (OR $2.305 ; 95 \%$ CI 1.053-5.046; $p=0.012$ ), as well as male
Table 4 Differences between patients with or without neurological disease

\begin{tabular}{lllr}
\hline Demographic and Clinical features & $\begin{array}{l}\text { Patients with } \\
\text { neurological disease } \\
(N=77)\end{array}$ & $\begin{array}{l}\text { Patients without } \\
\text { neurological disease } \\
(N=267)\end{array}$ & P value \\
\hline Severe infection - N (\%) & $51(66.2 \%)$ & $67(25.1 \%)$ & $<.001$ \\
Gender (males/females - \%) & $37 / 40(48.1 \% / 51.9 \%)$ & $167 / 100(62.5 \% / 37.5 \%)$ & 0.023 \\
Age (years) & $74.1 \pm 15.9(30-97)$ & $57.9 \pm 16.6(15-98)$ & $<.001$ \\
Time from symptoms onset (days) & $4.6 \pm 3.8(0-15)$ & $6.5 \pm 4.5(0-30)$ & 0.001 \\
Arterial Hypertension - N (\%) & $57(74.0 \%)$ & $101(37.8 \%)$ & $<.001$ \\
Neoplastic Diseases - N (\%) & $23(29.9 \%)$ & $26(9.7 \%)$ & $<.001$ \\
Diabetes - N (\%) & $12(15.6 \%)$ & $30(11.2 \%)$ & 0.305 \\
Chronic Obstructive Pulmonary Disease- $N(\%)$ & $13(16.9 \%)$ & $28(10.5 \%)$ & 0.127 \\
Moderate-to-severe renal failure- $N(\%)$ & $10(13.0 \%)$ & $8(3.0 \%)$ & 0.001 \\
Charlson comorbidity index & $4.3 \pm 2.9(0-16)$ & $1.3 \pm 1.8(0-11)$ & $<.001$ \\
Institutionalized patients- $N(\%)$ & $15(19.5 \%)$ & $4(1.5 \%)$ & $<.001$ \\
\hline
\end{tabular}

Results are reported as average \pm standard deviation (range) or absolute values (percentage), as appropriate. $p$ value: statistical comparison between the two groups 
Table 5 Univariate analysis of association between patients' characteristics and severe form of infection

\begin{tabular}{llc}
\hline Demographic and clinical features & OR $(95 \% \mathrm{CI})$ & $\begin{array}{c}\mathrm{P} \\
\text { value }\end{array}$ \\
\hline Male gender & $1.942(1.212-3.110)$ & 0.006 \\
Age & $1.076(1.057-1.095)$ & $<.001$ \\
Time from symptoms onset & $0.998(0.949-1.050)$ & 0.940 \\
Neurological disease & $5.855(3.387-10.122)$ & $<.001$ \\
Arterial hypertension & $4.236(2.631-6.821)$ & $<.001$ \\
Neoplastic disease & $4.575(2.413-8.672)$ & $<.001$ \\
Diabetes & $2.950(1.528-5.696)$ & 0.001 \\
Chronic obstructive pulmonary & $2.497(1.291-4.830)$ & 0.007 \\
$\quad$ disease & & \\
Moderate-to-severe renal failure & $5.835(2.231-10.088)$ & 0.001 \\
Charlson comorbidity index & $1.600(1.408-1.819)$ & $<.001$ \\
Institutionalization & $18.851(4.275-83.135)$ & $<.001$ \\
\hline
\end{tabular}

Results are reported as Odds Ratio (OR); 95\% Confidence Interval is reported in brackets. P-value: statistical significance

gender $(p=0.001)$, older age $(p=0.001)$, neoplastic diseases $(p=0.039)$, and arterial hypertension $(p=0.045)$.

After the ER admittance, patients affected by neurological diseases showed a lower rate of discharge at home ( $15.6 \%$ vs. $42.7 \%$; p < 0.001$)$, a higher rate of non-invasive mechanical respiratory support $(15.6 \%$ vs. $4.9 \% ; p=0.001)$ and intensive care indication $(14.3 \%$ vs. $4.5 \%$; $p=0.002)$. In contrast, they showed a similar rate of hospitalization without the need of mechanical respiratory support $(54.5 \%$ vs. $47.9 \%$; $p=0.307$ ).

After dividing the sample in patients without any comorbidities, patients with neurological disease without other comorbidities, patients with other comorbidities without neurological diseases, and patients with neurological disease and other comorbidities, the latter group showed the strongest association with a severe COVID-19 (OR 7.394; 95\% CI 1.840-29.704; $p=0.005)$, compared with patients with neurological (OR 2.332; 95\% CI 1.202-6.858; $p=0.035$ ) or non-neurological (OR 1.724; 95\% CI 1.100-3.790; $p=0.041$ ) comorbidity alone (Fig. 1b).

\section{Comparison of infection severity among different neurological diseases}

The association between neurological comorbidity and COVID-19 severity varied among the different neurological diseases (Table 6). Patients affected by cerebrovascular diseases and cognitive impairment showed a higher prevalence of severe infection, a lower rate of discharge at home and a higher rate of non-invasive mechanical ventilation or intensive care indication, significantly different from patients without neurological diseases $(p<0.001)$.
Conversely, patients affected by headache/facial pain, epilepsy, and peripheral neuropathy did not show any significant differences.

Both patients affected by multiple sclerosis and Parkinson disease (not shown in Table 6) suffered from severe COVID-19 that required hospitalization, without the need of mechanical respiratory support.

\section{Discussion}

In this study, we evaluated the prevalence of neurological pre-existing comorbidities in a large cohort of patients admitted to ER and diagnosed with COVID-19, estimating their association with infection severity. Over $20 \%$ of patients presented with neurological comorbidities, with cerebrovascular disease and cognitive impairment being the most frequent. Patients with neurological comorbidity showed an OR of 2.3 of suffering from severe COVID-19, even after including age and other clinical and demographic characteristics in the multivariate analysis. This association was stronger when patients suffering from a neurological condition in association with other comorbidities were compared to patients with isolated neurological or non-neurological diseases. Cerebrovascular diseases and cognitive impairment showed higher rate of severe infection and respiratory support indication, and lower rate of discharge at home.

To date, the incidence of new-onset neurological symptoms or syndromes associated with COVID-19 has been reported [3-8], but the description of the relationship between pre-existing neurological comorbidities and infection severity still lacks. A recent review by Herman and colleagues reported a pooled prevalence of neurological comorbidities of $8 \%$ among 22 reviewed studies (range 0-40\%), without conclusive data on the association with infection severity [12]. A recent study on inpatients from a neurological ward [11], showed worse clinical and functional outcomes, a more frequent use of high-flow oxygenation and antibiotic/antiviral treatments, longer hospitalization, and higher in-hospital mortality rate in patients with neurological diseases and COVID-19, compared to patients without infection; the vast majority of patients with COVID-19 were hospitalized for acute cerebrovascular events. Moreover, a meta-analysis reported that pre-existing cerebrovascular diseases could be an independent risk factor for COVID-19 [15]. In small-sample studies, cerebrovascular diseases have been associated with more frequent ICU admission [16] and with more severe forms of infection $[17,18]$. In addition, Du and colleagues reported a 2.4-fold higher mortality risk in patients with cerebrovascular or cardiovascular disease, without specifying the distinction between these two conditions [19]. 
Fig. 1 Multivariate analysis of association between patients' characteristics and severe form of infection. Odds ratios of associations between patients' characteristics and severe form of infection. In part $b$, values are adjusted for age and CCI, and patients without any comorbidities were used as reference group. CCI Charlson Comorbidity Index, COPD chronic obstructive pulmonary disease

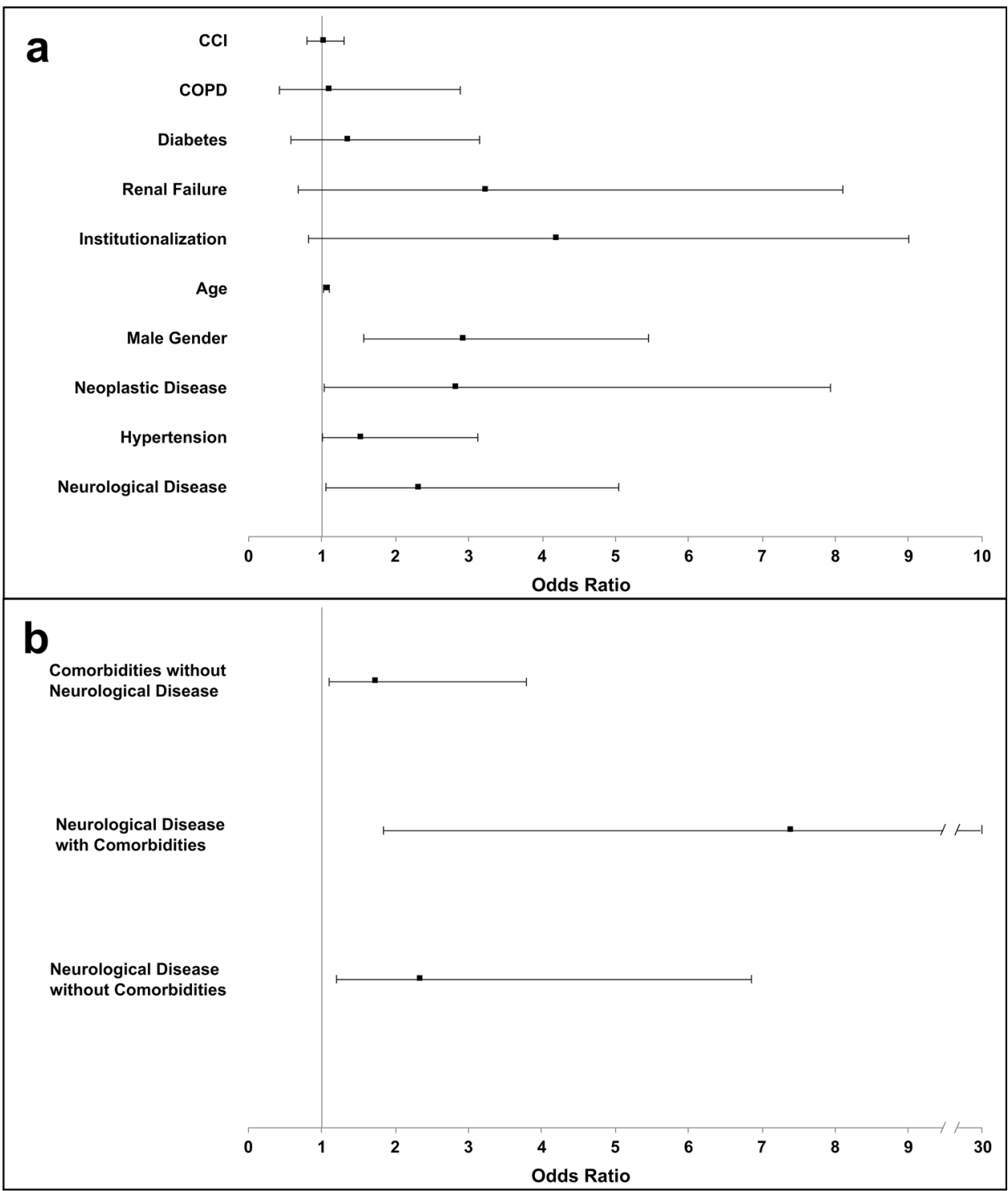

Table 6 Differences among neurological diseases

\begin{tabular}{|c|c|c|c|c|c|c|c|}
\hline & $\begin{array}{l}\text { Cerebro- } \\
\text { vascular } \\
(N=30)\end{array}$ & $\begin{array}{l}\text { Cognitive } \\
\text { impairment } \\
(N=25)\end{array}$ & $\begin{array}{l}\text { Headache } \\
(\mathrm{N}=11)\end{array}$ & $\begin{array}{l}\text { Epilepsy } \\
(\mathrm{N}=5)\end{array}$ & $\begin{array}{l}\mathrm{PNP} \\
(\mathrm{N}=4)\end{array}$ & $\begin{array}{l}\text { Patients without neuro- } \\
\text { logical disease }(\mathrm{N}=267)\end{array}$ & $p$ value \\
\hline Severe infection- $N(\%)$ & $20(66.7 \%)^{*}$ & $22(88.0 \%)^{*}$ & $3(27.3 \%)$ & $2(40.0 \%)$ & $2(50.0 \%)$ & $67(25.1 \%)$ & $<.001$ \\
\hline $\begin{array}{l}\text { Discharge from emergency room }-N \\
(\%)\end{array}$ & & & & & & & $<.001$ \\
\hline Home & $4(13.3 \%)^{*}$ & $0(0.0 \%)^{*}$ & $7(63.6 \%)$ & $1(20.0 \%)$ & $0(0.0 \%)$ & $114(42.7 \%)$ & \\
\hline $\begin{array}{l}\text { Internal Medicine Unit without mechan- } \\
\text { ical respiratory support }\end{array}$ & $16(53.3 \%)$ & $15(60.0 \%)$ & $3(27.3 \%)$ & $3(60.0 \%)$ & $3(75.0 \%)$ & $128(47.9 \%)$ & \\
\hline $\begin{array}{l}\text { Internal Medicine Unit with non-inva- } \\
\text { sive mechanical respiratory support }\end{array}$ & $3(10.0 \%)$ & $7(28.0 \%)^{*}$ & $1(9.1 \%)$ & $1(20.0 \%)$ & $0(0.0 \%)$ & $13(4.9 \%)$ & \\
\hline Intensive Care Unit & $7(23.3 \%)^{*}$ & $3(12.0 \%)$ & $0(0.0 \%)$ & $0(0.0 \%)$ & $1(25.0 \%)$ & $12(4.5 \%)$ & \\
\hline
\end{tabular}

Results are reported as absolute values (percentage). $p$-value: statistical comparison among groups. *: significant difference vs. Patients without neurological disease $(p<0.001)$. Headache: migraine or chronic tension-type headache or trigeminal neuralgia 
Our data show that patients with pre-existing cerebrovascular diseases are more frequently hospitalized, needing ICU admission in over $20 \%$ of cases, and present with a severe infection in two-thirds of cases. We observed similar findings in cognitively impaired/demented patients. A recent paper reported a prevalence of $6.8 \%$ of dementia in a sample of 355 Italian patients who died with COVID-19, without assessing the association with infection severity [20].

In our samples, we observed that patients suffering from neurological diseases were older, more affected by other comorbidities, and more institutionalized. Age is a frequently reported risk factor for severe COVID-19 [16, 19], as well as hypertension [21], diabetes [21, 22], neoplastic diseases [21, 23], COPD [21], and institutionalization [24]. These observations were confirmed in our study. The multivariate analysis showed that even correcting for age, institutionalization, and comorbidities, the presence of neurological diseases seems to be independently associated with a more severe form of infection. The strongest association with severe COVID-19 was observed in patients with both neurological diseases and other comorbidities. This finding is consistent with the results reported by Guan and colleagues, who found hazard ratios for worse infection outcomes as higher as the number of concomitant diseases increases [21]. On the other hand, institutionalization, diabetes, renal failure, and COPD showed a high association with infection severity only in the univariate analysis, but not in the multivariate analysis.

The higher prevalence of severe COVID-19 in patients affected by neurological diseases is probably multifactorial. The intrinsic frailty of chronic and often degenerative conditions, the older age, and the higher comorbidity burden observed in these patients could certainly explain this association [25]. Patients affected by neurological diseases could present a lower ability to compensate for COVID-19, resulting in more severe infection and higher need of ER assistance. Moreover, the potential neurotropism of SARS$\mathrm{CoV}-2$, with a possible detrimental effect on pre-existing neurological diseases, should also be taken into account [9, 10], as already postulated during the SARS-CoV epidemic in 2003 [26]. While waiting for more accurate pathological evidences, our data underline that patients with neurological diseases, in particular when associated with other comorbidities, represent a population at high risk for severe COVID-19, needing a careful health surveillance.

Our study presents with some limitations. First, the single-center design, which partially restricts the generalizability of our findings. Second, only a minority of patients suffered from neurological diseases without other comorbidities, limiting the conclusions on the association between neurological pathology alone and severe COVID-19. Third, the observations on the prevalence of each neurological disorder, and thus the ability to discriminate disease-specific associations with COVID-19, are limited by two factors: a) the evaluation of patients admitted to ER, which could represent a selection bias towards older people, and b) the lack of adjustments for the relative prevalence of each neurological disease in the general population.

In conclusion, our study reports the prevalence of different neurological diseases in a large cohort of patients with COVID-19, assessing their association with the infection severity. In our sample, patients with pre-existing neurological diseases showed a significantly higher risk for severe infection, in particular when associated with other comorbidities, suggesting that this population deserves a thorough evaluation since the earliest phases of overt or suspected COVID-19. Finally, our findings suggest a particular attention in targeting patients with neurological diseases for proactive viral screening.

Acknowledgments Open access funding provided by Universit ̃̃ degli Studi di Torino within the CRUI-CARE Agreement. The Authors would like to express their gratitude to all the clinicians and Front Line staff engaged in the care of COVID-19 patients, and to General and Health Management Department (G. La Valle, L. Angelone, V. Alpe. A. Scarmozzino) of "Città della Salute e della Scienza Hospital", Turin, for the great work carried out during this sanitary emergency.

Authorship statement AR: conception and design of the study; analysis and interpretation of data; writing the first draft. RB: acquisition, analysis and interpretation of data; critical revision for important intellectual content. GI: acquisition, analysis and interpretation of data; critical revision for important intellectual content. GC: interpretation of data, revising the manuscript for content. FR: interpretation of data, revising the manuscript for content. CAA: interpretation of data, revising the manuscript for content. MB: interpretation of data, revising the manuscript for content. BF: interpretation of data, revising the manuscript for content. EM: interpretation of data, revising the manuscript for content. EM: interpretation of data, revising the manuscript for content. MGR: interpretation of data, revising the manuscript for content. GV: interpretation of data, revising the manuscript for content. MZ: interpretation of data, revising the manuscript for content. LL: conception and design of the study; analysis and interpretation of data; revising the manuscript for content. All the co-authors listed above gave their final approval of this manuscript version. All the co-authors agree to be accountable for all aspects of the work in ensuring that questions related to the accuracy or integrity of any part of the work are appropriately investigated and resolved.

Financial disclosures Dr Romagnolo has received grant support and speaker honoraria from AbbVie, speaker honoraria from Chiesi Farmaceutici and travel grants from Lusofarmaco, Chiesi Farmaceutici, Medtronic, and UCB Pharma.

Data access and responsibility statement A. Romagnolo had full access to all the data in the study and takes responsibility for the integrity of the data and the accuracy of the data analysis.

\section{Compliance with ethical standards}

Conflicts of interest Dr Balestrino has no financial conflicts to disclose. Dr Imbalzano has no financial conflicts to disclose. Dr Ciccone has no financial conflicts to disclose. Dr Riccardini has no financial 
conflicts to disclose. Dr Artusi has received travel grants from Zambon and Abbvie. Dr Bozzali has no financial conflicts to disclose. Dr Ferrero has no financial conflicts to disclose. Dr Montalenti has no financial conflicts to disclose. Dr Montanaro has received travel grant from Ralpharma. Dr Rizzone has received grant support and speaker honoraria from Medtronic and UCB. Dr Vaula has no financial conflicts to disclose. Dr Zibetti has received honoraria from Medtronic, Zambon Pharma and AbbVie. Dr. Lopiano has received honoraria for lecturing and travel grants from Medtronic, UCB Pharma, and AbbVie.

Ethics approval This study received approval from the ethical standards committee on human experimentation (Comitato Etico Interaziendale AOU Città della Salute e della Scienza di Torino, AO Ordine Mauriziano di Torino, ASL Città di Torino; Protocol number 00172/2020, approved May 5th, 2020), and patients gave their written informed consent. The authors declare that they acted in accordance with the ethical standards laid down in the 1964 Declaration of Helsinki.

Open Access This article is licensed under a Creative Commons Attribution 4.0 International License, which permits use, sharing, adaptation, distribution and reproduction in any medium or format, as long as you give appropriate credit to the original author(s) and the source, provide a link to the Creative Commons licence, and indicate if changes were made. The images or other third party material in this article are included in the article's Creative Commons licence, unless indicated otherwise in a credit line to the material. If material is not included in the article's Creative Commons licence and your intended use is not permitted by statutory regulation or exceeds the permitted use, you will need to obtain permission directly from the copyright holder. To view a copy of this licence, visit http://creativecommons.org/licenses/by/4.0/.

\section{References}

1. COVID-19 situation reports. World Health Organization (WHO). Available at: https://www.who.int/emergencies/diseases/novel -coronavirus-2019/situation-reports. Accesssed May 15, 2020

2. Coronaviridae Study Group of the International Committee on Taxonomy of Viruses (2020) The species Severe acute respiratory syndrome-related coronavirus: classifying 2019-nCoV and naming it SARS-CoV-2. Nat Microbiol 5:536-544

3. Mao L, Jin H, Wang M et al (2020) Neurologic manifestations of hospitalized patients with coronavirus disease 2019 in Wuhan, China. JAMA Neurol 77:1-9

4. Beyrouti R, Adams ME, Benjamin L et al (2020) Characteristics of ischaemic stroke associated with COVID-19. J Neurol Neurosurg Psychiatry 91:889-891

5. Lechien JR, Chiesa-Estomba CM, De Siati DR et al (2020) Olfactory and gustatory dysfunctions as a clinical presentation of mild-to-moderate forms of the coronavirus disease (COVID19): a multicenter European study. Eur Arch Otorhinolaryngol 277:2251-2261

6. Zhao H, Shen D, Zhou H et al (2020) Guillain-Barré syndrome associated with SARS-CoV-2 infection: causality or coincidence? Lancet Neurol 19:383-384

7. Pilotto A, Odolini S, Masciocchi S et al (2020) Steroid-responsive encephalitis in Covid-19 disease. Ann Neurol. https://doi. org/10.1002/ana.25783

8. Jin $\mathrm{H}$, Hong $\mathrm{C}$, Chen $\mathrm{S}$ et al (2020) Consensus for prevention and management of coronavirus disease 2019 (COVID-19) for neurologists. Stroke Vasc Neurol 5:146-151
9. Baig AM, Khaleeq A, Ali U, Syeda H (2020) Evidence of the COVID-19 virus targeting the CNS: tissue distribution, host-virus interaction, and proposed neurotropic mechanisms. ACS Chem Neurosci 11:995-998

10. Li Y-C, Bai W-Z, Hashikawa T (2020) The neuroinvasive potential of SARS-CoV2 may play a role in the respiratory failure of COVID-19 patients. J Med Virol 92:552-555

11. Benussi A, Pilotto A, Premi E et al (2020) Clinical characteristics and outcomes of inpatients with neurological disease and COVID19. Neurology. https://doi.org/10.1212/WNL.0000000000009848

12. Herman C, Mayer K, Sarwal A (2020) Scoping review of prevalence of neurologic comorbidities in patients hospitalized for COVID-19. Neurology 95:77-84

13. Charlson ME, Pompei P, Ales KL et al (1987) A new method of classifying prognostic comorbidity in longitudinal studies: development and validation. J Chronic Dis 40:373-383

14. Metlay JP, Waterer GW, Long AC et al (2019) Diagnosis and Treatment of Adults with Community-acquired Pneumonia. An Official Clinical Practice Guideline of the American Thoracic Society and Infectious Diseases Society of America. Am J Respir Crit Care Med 200:e45-e67

15. Wang B, Li R, Lu Z et al (2020) Does comorbidity increase the risk of patients with COVID-19: evidence from meta-analysis. Aging (Albany NY) 12:6049-6057

16. Wang D, Hu B, Hu C et al (2020) Clinical characteristics of 138 hospitalized patients with 2019 novel coronavirus-infected pneumonia in Wuhan, China. JAMA 323:1061-1069

17. Mo P, Xing Y, Xiao Y et al (2020) Clinical characteristics of refractory COVID-19 pneumonia in Wuhan, China. Clin Infect Dis. https://doi.org/10.1093/cid/ciaa270

18. Aggarwal G, Lippi G, Michael Henry B (2020) Cerebrovascular disease is associated with an increased disease severity in patients with Coronavirus Disease 2019 (COVID-19): a pooled analysis of published literature. Int J Stroke 15:385-389

19. Du R-H, Liang L-R, Yang C-Q et al (2020) Predictors of mortality for patients with COVID-19 pneumonia caused by SARS-CoV-2: a prospective cohort study. Eur Respir J 55:2000524

20. Onder G, Rezza G, Brusaferro S (2020) Case-fatality rate and characteristics of patients dying in relation to COVID-19 in Italy. JAMA 323:1775-1776

21. Guan W-J, Liang W-H, Zhao Y et al (2020) Comorbidity and its impact on 1590 patients with Covid-19 in China: a nationwide analysis. Eur Respir J 55:2000547

22. Yan Y, Yang Y, Wang F et al (2020) Clinical characteristics and outcomes of patients with severe covid-19 with diabetes. BMJ Open Diabetes Res Care 8:e001343

23. Liang W, Guan W, Chen R et al (2020) Cancer patients in SARSCoV-2 infection: a nationwide analysis in China. Lancet Oncol 21:335-337

24. Kim S-W, Su K-P (2020) Using psychoneuroimmunity against COVID-19. Brain Behav Immun 87:4-5

25. Roca F, Lang P-O, Chassagne P (2019) Chronic neurological disorders and related comorbidities: role of age-associated physiological changes. Handb Clin Neurol 167:105-122

26. Netland J, Meyerholz DK, Moore S, Cassell M, Perlman S (2008) Severe acute respiratory syndrome coronavirus infection causes neuronal death in the absence of encephalitis in mice transgenic for human ACE2. J Virol 82:7264-7275 